\title{
Relação entre ordem de nascimento e interesses vocacionais
}

\author{
Relationship between order of birth \\ and vocational interests
}

Mauro de Oliveira MAGALHÃES ${ }^{1}$

\begin{abstract}
Resumo
As teorias do desenvolvimento humano enfatizam a importância das experiências infantis na formação da personalidade. Acredita-se que o papel assumido pela criança na família tem implicações no comportamento adulto posterior. Adler propôs que a ordem de nascimento tem influência significativa no comportamento vocacional, e pesquisas recentes têm apoiado esta idéia. Este estudo teve por objetivo investigar diferenças de interesses vocacionais entre as seguintes categorias de ordem de nascimento: filhos únicos, primogênitos, 'do meio' e caçulas. Participaram 435 estudantes universitários de ambos os sexos, com idades entre 17 e 56 anos (Média=26,9). Os sujeitos responderam a uma medida de interesses vocacionais e a um questionário demográfico para definição da categoria de ordem de nascimento. Os escores de interesses foram comparados por análises de variância. Os resultados revelaram que filhos únicos têm maior interesse em investigação científica do que filhos 'do meio', e menor interesse empreendedor do que primogênitos. Os filhos 'do meio' revelaram interesse social mais elevado do que filhos únicos e primogênitos. Concluiu-se que filhos únicos, primogênitos, 'do meio' e caçulas desenvolvem processos vocacionais distintos, e recomenda-se o uso de informações sobre ordem de nascimento no aconselhamento vocacional.
\end{abstract}

Unitermos: Escolha profissional. Ordem de nascimento. Orientação profissional.

\begin{abstract}
Theories of human development emphasize the importance of childhood experiences in the formation of personality. It is believed that the role assumed by the child within the family has implications for his later adult life. Adler proposed that the order of birth significantly influences vocational behavior and recent research has provided support for this idea. The present study has the objective of investigating differences in vocational interests among the following categories, by order of birth: only children, firstborn, middle children and lastborn. The subjects comprised 435 college students of both sexes with ages ranging from 17 to 56 years (Mean=26.9). The subjects answered measures of vocational interests and a brief demographic questionnaire to define the order of birth category. The scores for their interests were compared by means of variance analysis. The results revealed that only children showed greater interest in scientific investigation than middle children and less entrepreneurial interest than the firstborn; middle children displayed greater social interest than only children and firstborn. The results suggest that individuals develop distinct vocational processes depending on the order of birth, and therefore the use of order of birth information is recommended for vocational counseling.
\end{abstract}

Uniterms: Occupacional choice. Birth order. Occupational guidance.

$\cot$

1 Universidade Luterana do Brasil, Curso de Psicologia. Av. Farroupilha, 8001, São Luís, 92450-900, Canoas, RS, Brasil. Correspondência para/Correspondence to: E-mail:<mauro.m@terra.com.br>. 
Em qualquer circunstância que um número de pessoas venha a conviver continuamente, diferenças nas funções desempenhadas por cada um na vida comum se manifestam. Estas diferenças podem ocorrer nas tarefas ou contribuições trazidas por cada membro à coletividade. A ciência social cunhou o termo papel para designar esta especialização da função individual no grupo. Na família, ela ocorre naturalmente. Sendo assim, já nos primeiros anos da vida, a criança assume um papel mais ou menos definido, o qual tende a ser reconhecido pelos demais membros da família e, a seguir, por outras pessoas do seu meio social mais amplo. Obviamente, este papel irá afetar o desenvolvimento do indivíduo e refletir no seu comportamento quando adulto. Na vida familiar, a divisão de papéis será influenciada por variações de sexo, ordem de nascimento, idade e diferenças de idade, assim como por características inatas (Bradley \& Mims, 1992). A ordem de nascimento foi citada por teóricos da personalidade, desde as idéias de Adler (1912/1954), como um fator relevante para o desenvolvimento da personalidade.

Muitas pesquisas têm investigado a relação entre a posição ordinal na genitura familiar e inúmeras variáveis, tais como inteligência (Zajonc, 2001), criatividade (Baer, Oldham, Hollingshead \& Jacobsohn, 2005), hipocondria (Skinner, 1997), feedback parental (Claxton, 1994), status profissional (Terry, 1989), entre outras. A proliferação de pesquisas sobre o tema levou Zajonc e Mullally (1997) a afirmar que "a ordem de nascimento tem sido rapidamente restabelecida como um fator relevante em psicologia" (p.698).

Este estudo investigou a relação entre ordem de nascimento e interesses vocacionais. Outras pesquisas já examinaram esta questão (Bryant, 1987; Leong, Hartung, Goh \& Gaylor, 2001), assim como há trabalhos que enfatizaram a utilidade do entendimento da psicodinâmica do grupo de irmãos para o aconselhamento vocacional (Bradley, 1984; Bradley \& Mims, 1992).

A teoria vocacional adleriana postula que variáveis familiares têm influência marcante no desenvolvimento da carreira e no comportamento vocacional (Savickas, 1998; Watkins, 1984). Watkins (1984) propôs que os pais apresentam demandas e expectativas diferentes para as crianças que ocupam posições diversas na ordem de nascimento (primogênitos, caçulas etc.),

204 que irão gerar conseqüências vocacionais distintas.
Comportamentos relacionados ao papel de trabalho e autoconceitos implicados, estilo interpessoal de trabalho, interesses vocacionais e valores são aspectos influenciados pelo contexto familiar. Savickas (1998) reforçou esta idéia, salientando que a ordem de nascimento interfere significativamente no estilo de carreira e nos interesses vocacionais do indivíduo.

O trabalho pioneiro de Galton (apud Sulloway, 1999a), realizado no final do século XIX, salientou a relação entre ordem de nascimento e status profissional, informando que cientistas eminentes eram, mais freqüentemente, primogênitos ou filhos únicos. Vários estudos corroboraram esta conclusão. Os primogênitos são predominantes entre os ganhadores do prêmio Nobel (Clark \& Rice, 1982), compositores clássicos (Schubert, Wagner \& Schubert, 1977), e mesmo entre psicólogos eminentes (Terry, 1989). Recentemente, a pesquisa de Galton foi reconsiderada por Sulloway (1999a), que trouxe evidências convincentes de que os nascidos mais tarde são, na realidade, mais revolucionários e inovadores do que os primeiros, pois lideraram 23 das 28 revoluções científicas nos últimos 300 anos (Sulloway, 1999a). Ainda outros estudos mostraram que os caçulas tendem a se tornar líderes revolucionários e são, na verdade, mais criativos do que seus irmãos primogênitos (Simonton, 1999a, 1999b; Sulloway, 1999a, 1999b). Portanto, as pesquisas sugerem que a relação entre a posição de primogênito e a eminência científica é restrita a um certo tipo de realização acadêmica.

Segundo a teoria de Adler, o segundo filho desenvolverá mais os seus talentos do que o primogênito, pois sua posição na família o induz à competição com aquele que defende a primeira posição (Ansbacher \& Ansbacher, 1956). De acordo com esta visão, o primogênito define o ritmo para o segundo, que é motivado a alcançá-lo. Adler (1929/1969) considerou que o primogênito seria um bom supervisor e parceiro de trabalho, mas preocupado demais com regras para fazer contribuições inovadoras. Em contraste, o mais jovem teria tendências revolucionárias, pois, segundo o autor, "ele está muito mais inclinado a acreditar, acertadamente ou não, que não existe poder no mundo que não possa ser destituído" (Ansbacher \& Ansbacher, 1956, p.380). Enfim, observar uma maior freqüência de primogênitos envolvidos com ciência e um maior número de caçulas 
liderando revoluções científicas não resulta necessariamente em contradição, pois a eminência científica pode ser alcançada com ou sem rebelião contra o paradigma vigente.

A maior parte dos estudos sobre ordem de nascimento e status profissional foi feita com homens. Por exemplo, um número maior do que o esperado de homens primogênitos foi encontrado na advocacia (Very \& Prull, 1970), na medicina (Watkins \& Savickas, 1990), ou dedicados à pesquisa científica (Helson \& Crutchfield, 1970; Roe, 1953). Recentemente, surgiram evidências de que esta relação também é significativa para as mulheres. Bohmer e Sitton (1993) compararam a ordem de nascimento e o número de irmãos de 201 mulheres consideradas expoentes na sua área profissional. Em contraste com os estudos sobre os homens, as análises revelaram que mulheres caçulas selecionaram carreiras científicas e se destacaram mais freqüentemente do que as demais. Ora, se Adler (1929/1969) e Sulloway (1999a) estão corretos na caracterização dos caçulas como revolucionários, no caso feminino esta propensão pode estar sendo expressa pela escolha de carreiras não tradicionais para mulheres, tais como a ciência. A não tradicionalidade da preferência por carreiras científicas em mulheres ainda pode ser verificada em pesquisas recentes (Magalhães, Balbinotti \& Fisher, 2004). Por outro lado, em pesquisa anterior, Melillo (1983) encontrou, em uma amostra de 174 acadêmicas doutoras, uma proporção inesperadamente elevada de primogênitas e filhas únicas.

A pesquisa sobre a relação entre ordem de nascimento e carreira profissional investigou predominantemente a distribuição dos indivíduos entre ocupações (Leong et al., 2001; Watkins \& Savickas, 1990). Poucos estudos investigaram a relação entre ordem de nascimento e interesses vocacionais. Este tipo de investigação é relevante e necessário, uma vez que os interesses nem sempre se traduzem direta e automaticamente em escolhas ocupacionais. Também há carência de pesquisas que articulem o constructo ordem de nascimento com teorias consagradas da psicologia vocacional, tais como as de Holland (1997) e Super (Super, Savickas \& Super, 1996).

Uma exceção foi a pesquisa de Leong et al. (2001), que investigou a relação entre ordem de nascimento e interesses vocacionais medidos pelo modelo de Holland (1997). Leong et al. (2001) encontraram, em uma amostra de estudantes de medicina, que caçulas possuem interesses artísticos e realistas mais elevados do que primogênitos, e que filhos únicos apresentam maior interesse investigativo do que os demais. Leong et al. (2001) consideraram que a tendência à superproteção dos filhos únicos pode resultar na preferência destes por assuntos acadêmicos (interesse investigativo), ao invés de atividades físicas e ao ar livre (interesse realista). Além disto, sugeriram que os pais talvez encorajem filhos únicos ou primogênitos a investir em carreiras tradicionais e de maior prestígio e, deste modo, as crianças mais jovens acabam por mostrar interesse por carreiras artísticas. Na medida em que outras crianças nascem, os pais tendem a ser mais abertos e liberais, e isto pode permitir que crianças mais jovens assumam maiores riscos. Por exemplo, se o primogênito ou filho único decide ser um poeta, isto pode preocupar os pais. Mas se o quarto filho o faz, eles talvez não se importem tanto (Leong et al., 2001). Estas idéias corroboram a proposição de que os caçulas tendem a ser mais ousados e assumir mais riscos (Adler, 1912/1954; Sulloway, 1999a). Neste sentido, outras pesquisas encontraram que os nascidos por último tendem a escolher esportes mais perigosos do que primogênitos, tanto em amostras femininas (Sohl \&Yusuff, 1991) quanto masculinas (Nisbett, 1968).

Por fim, em um estudo com mulheres universitárias, Bryant (1987) comparou interesses de primogênitas e caçulas, encontrando que as primeiras preferem trabalhar com independência, manifestando o desejo por uma carreira na qual o indivíduo seja autônomo e/ou esteja em uma posição de dirigir as atividades de outros. E, de acordo com o autor, são mais propensas a obter sucesso em carreiras acadêmicas. De um modo geral, as pesquisas apóiam a teoria adleriana, ao indicarem que a ordem de nascimento e a configuração familiar influenciam o comportamento vocacional (Leong et al., 2001; Watkins \& Savickas, 1990; Watts \& Engels, 1995).

Holland (1997) propôs uma tipologia de interesses vocacionais que tem dominado o cenário das pesquisas sobre o tema nos últimos 20 anos (Anastasi \& Urbina, 2000). A teoria não será abordada em detalhes aqui, mas uma extensa revisão sobre o assunto pode ser encontrada em Magalhães (2005). De acordo com o 
modelo, em nossa cultura, os interesses podem ser de seis tipos: realista (R), investigativo (I), artístico (A), social (S), empreendedor (E) e convencional (C). Para o autor, os interesses vocacionais são uma expressão da personalidade, e indivíduos de uma mesma ocupação possuem personalidades similares. $O$ interesse realista significa a preferência por atividades práticas, objetivas e concretas, tais como o trabalho com máquinas e animais. $O$ interesse investigativo se refere a atividades voltadas para conteúdos abstratos e teóricos, como a ciência. Indivíduos de interesses predominantemente realistas e investigativos evitam atividades que envolvam o contato interpessoal mais intenso, tais como ensino, persuasão ou vendas, que, por sua vez, são preferidas por aqueles de interesses mais sociais e empreendedores. $\mathrm{O}$ interesse social significa o desejo de assistir e auxiliar pessoas com dificuldades. O empreendedor caracteriza a preferência por atividades de persuasão e liderança, com a valorização de conquistas materiais e status social. O interesse artístico revela a vontade de expressar sentimentos por meio de produções estéticas e criativas. Em contraste, o interesse convencional se refere ao gosto pela rotina, organização, estrutura e previsibilidade.

Holland (1985, 1997), que formulou a sua teoria em bases adlerianas, também propôs que as personalidades vocacionais começam a se formar na infância, em função da dinâmica familiar, dentro da qual "as crianças, até certo ponto, criam o seu próprio ambiente através das suas demandas sobre os pais e através da maneira pela qual os pais reagem e são influenciados pelas crianças" (Holland, 1985, p.16). Embora as teorias de Adler (1912/1954) e Holland (1997) destaquem a importância da constelação familiar no comportamento vocacional, poucos estudos têm buscado conectar estas abordagens. Esta pesquisa verificou se o constructo ordem de nascimento, articulado por Adler, se relaciona significativamente com o modelo de personalidades vocacionais proposto por Holland (1997). A questão que guiou esta investigação foi examinar se existem diferenças significativas de interesse vocacional entre filhos únicos, primogênitos, 'do meio'e caçulas. As expectativas lançadas foram que filhos únicos e primogênitos apresentassem maior interesse do tipo investigativo do que os demais, e que caçulas mostrassem mais interesse 206 artístico.

\section{Método}

\section{Participantes}

Participaram do estudo 435 estudantes universitários (196 homens e 239 mulheres), com idades entre 17 e 56 anos (Média - M=26,9; Desvio-padrão - DP=7,7) de diversos cursos: engenharia elétrica (60), secretariado executivo (47), serviço social (70), psicologia (41), teologia (23), informática (48), administração de empresas (63), química (41), matemática (32).

\section{Instrumentos}

Os sujeitos responderam à Escala de Atividades do SelfDirected Search (Holland, 1997), a fim de medir os interesses vocacionais. A Escala de Atividades é constituída de 66 itens que descrevem atividades de trabalho. Os itens são agrupados em 11 itens realistas, 11 investigativos, 11 artísticos, 11 sociais, 11 empreendedores e 11 convencionais. Os sujeitos assinalaram o quanto gostavam ou não destas atividades em uma escala Likert de cinco pontos. Esta escala mostrou qualidades psicométricas satisfatórias em amostras brasileiras (Balbinotti, Magalhães, Callegari \& Fonini, 2004). Também foi aplicado um breve questionário demográfico com questões relativas à estrutura familiar, ordem de nascimento e idades de cada membro do grupo de irmãos.

A coleta de dados foi realizada em sala de aula, sendo que os professores, oportunamente e com autorização da direção do respectivo curso, disponibilizaram 60 minutos para o procedimento. Os alunos foram convidados a participar da pesquisa e, aqueles que concordaram, assinaram o Termo de Consentimento Livre e Esclarecido.

\section{Procedimentos}

Os sujeitos foram agrupados de acordo com a ordem de nascimento, nas seguintes categorias: primogênitos, únicos, 'do meio'e caçulas. O primogênito foi definido como a primeira criança a nascer da união dos seus pais; o caçula como a última a nascer; o "do meio" foi aquele com irmãos mais velhos e mais jovens, e o filho único foi aquele que nasceu e viveu sem a companhia de outras crianças na mesma residência. 
Foram calculadas as diferenças de idade entre o sujeito e o irmão imediatamente mais velho e/ou mais jovem. No caso de uma diferença maior do que seis anos, seguindo a sugestão da literatura (Bohmer \& Sitton, 1993; Bryant, 1987), o sujeito foi excluído da amostra, para evitar imprecisão na definição da posição ordinal. Indivíduos com irmãos gêmeos, adotivos ou que informaram ser provenientes de famílias reconstituídas e que conviveram, quando crianças, com irmãos não-consangüíneos, também foram excluídos por este motivo. As diferenças de interesse vocacional entre as categorias de ordem de nascimento foram analisadas por meio de provas de análise de variância.

\section{Resultados}

A Tabela 1 apresenta os resultados da estatística descritiva dos escores das escalas de interesse vocacional em cada categoria de ordem de nascimento.

As análises de variância revelaram diferenças significativas entre os grupos nos interesses social $[F(3$, $419=3,16, p<0,05)]$, empreendedor $[F(3,418=3,99, p<0,01)]$ e investigativo $[F(3,417=3,39, p<0,05)]$. Nos testes post hoc (Tukey), os filhos "do meio" revelaram escores mais elevados em interesse social do que únicos e primogênitos $(p<0,05)$. Filhos únicos mostraram interesse investigativo mais elevado do que filhos "do meio", e primogênitos apresentaram interesse empreendedor mais elevado do que filhos únicos $(p<0,05)$.

\section{Discussão}

A diferença encontrada entre filhos únicos e "do meio" quanto ao interesse investigativo corrobora hipó- teses derivadas da literatura. $O$ interesse investigativo se refere à preferência por atividades de reflexão e pesquisa científica, o gosto por conteúdos abstratos e teóricos. A associação entre filhos únicos e dedicação à ciência foi salientada em pesquisas anteriores (Bryant, 1987; Helson \& Crutchfield, 1970; Roe, 1953). Porém, estes estudos não haviam verificado esta relação a partir da avaliação do interesse vocacional. Assim como na pesquisa de Leong et al. (2001), os resultados encontrados ratificam que filhos únicos tendem a preferir atividades investigativas e, portanto, a serem encontrados mais freqüentemente do que os demais em profissões nas quais há predomínio deste tipo de atividade.

Os autores sugeriram que filhos únicos tendem a receber cuidados especiais e ser o alvo de expectativas parentais mais elevadas quanto à realização e ao status profissional. Sendo assim, acabam por se dedicar mais aos estudos. Esta dedicação acadêmica também os afasta de atividades potencialmente perigosas que possam preocupar os pais que, geralmente receosos de que o seu único descendente venha a se ferir, tendem a controlar excessivamente o seu comportamento. Estas preocupações parentais também podem atingir aos primogênitos.

No estudo aqui descrito, os escores mais elevados dos primogênitos no interesse empreendedor, em comparação com filhos únicos, sugerem que o papel de líder e responsável pelos demais parece ter maior impacto em seu comportamento vocacional. Neste sentido, de acordo com Sulloway (1999a), o primogênito tende a ser mais responsável, conservador, ambicioso, planejador, assertivo e com disposição para a liderança. Estas características correspondem à descrição de Holland (1997) do indivíduo com interesse predominantemente empreendedor. Além disto, alguns auto-

Tabela 1. Médias (M) e desvios-padrão (DP) dos escores de interesse vocacional e valores de " $n$ " em cada ordem de nascimento.

\begin{tabular}{|c|c|c|c|c|c|c|c|c|c|c|c|c|c|c|c|}
\hline \multirow{3}{*}{$\begin{array}{l}\text { Interesse } \\
\text { vocacional }\end{array}$} & \multicolumn{15}{|c|}{ Posição na ordem de nascimento } \\
\hline & \multicolumn{3}{|c|}{ Filho único } & \multicolumn{3}{|c|}{ Primogênito } & \multicolumn{3}{|c|}{ "Do meio" } & \multicolumn{3}{|c|}{ Caçula } & \multicolumn{3}{|c|}{ Total } \\
\hline & $n$ & $M$ & DP & $\mathrm{n}$ & $M$ & DP & $\mathrm{n}$ & M & DP & $n$ & $M$ & DP & $\mathrm{n}$ & $M$ & DP \\
\hline Realista & 32 & 24,91 & 10,29 & 120 & 28,29 & 10,10 & 162 & 26,74 & 9,64 & 109 & 27,29 & 11,47 & 423 & 27,18 & 10,32 \\
\hline Investigativo & 32 & 34,28 & 8,07 & 119 & 32,14 & 9,68 & 161 & 29,42 & 10,15 & 109 & 29,80 & 10,42 & 421 & 30,66 & 10,04 \\
\hline Artístico & 32 & 30,16 & 9,84 & 120 & 30,16 & 11,57 & 161 & 30,91 & 11,46 & 108 & 30,44 & 10,98 & 421 & 30,52 & 11,22 \\
\hline Social & 32 & 33,13 & 9,97 & 120 & 33,93 & 11,43 & 162 & 37,33 & 10,30 & 109 & 34,82 & 10,20 & 423 & 35,40 & 10,67 \\
\hline Empreendedor & 32 & 31,28 & 10,68 & 119 & 38,36 & 9,48 & 162 & 37,67 & 11,10 & 109 & 37,37 & 10,70 & 422 & 37,50 & 10,63 \\
\hline Convencional & 32 & 24,19 & 9,70 & 120 & 27,38 & 11,01 & 162 & 27,44 & 11,34 & 109 & 25,41 & 11,05 & 423 & 26,66 & 11,07 \\
\hline
\end{tabular}


res sugeriram que o sentimento de ter sido destronado pelos mais jovens, após ter sido o centro das atenções, pode levar o primogênito a buscar o sentimento de pertencer com pessoas e grupos fora do ambiente familiar (Dreikurs, 1963; Fakouri \& Hafner, 1984). Sugere-se que, por ocasião do destronamento, uma decepção com os vínculos primários possa levar estes indivíduos a focarem sua atenção no meio social mais amplo, e a desenvolverem atitudes de independência e espírito aventureiro, associadas com o desejo de mostrar que são capazes de encontrar um lugar fora da família. Esta linha de movimento parece descrever a trajetória de pessoas empreendedoras. É uma hipótese que aguarda investigação.

No modelo de Holland (1997), o interesse investigativo foi descrito como oposto ao interesse empreendedor, sendo esperado que indivíduos com escores elevados em uma destas inclinações vocacionais apresentem escores inferiores na outra. Nesta perspectiva, não é surpresa observar escores inferiores de interesse empreendedor em indivíduos com escores elevados na inclinação investigativa, tal como se apresenta o grupo de filhos únicos.

Os sujeitos classificados como filhos "do meio" revelaram escores mais elevados de interesse social do que primogênitos e filhos únicos. Este resultado é coerente com a descrição de Adler (1929/1969; Ansbacher \& Ansbacher, 1956), na qual estas crianças foram caracterizadas como 'espremidas' entre irmãos mais velhos e mais jovens, e com dificuldade para encontrar o seu lugar na família. Sendo assim, acabam por desenvolver estratégias de cooperação para negociar o seu espaço no ambiente familiar. Portanto, a partir desta habilidade para assumir o papel de um diplomata, podem vir a desenvolver o interesse por atividades de assistência social. Por outro lado, primogênitos não tendem a desenvolver tais habilidades de cooperação, sendo mais dominadores e agressivos, pois desde o nascimento ocupam uma posição de poder e prestígio no grupo e lutam para preservá-la. E filhos únicos nunca foram chamados a dividir as atenções parentais com outros irmãos.

A maior liberdade e tolerância supostamente dispensada a filhos caçulas não se traduziram em interesse artístico mais elevado, conforme expectativas derivadas das idéias de Adler (1969/1929) e Sulloway (1999b). Talvez as características distintivas de filhos caçulas, tais como a rebeldia (Sulloway, 1999a), possam não ter representatividade no modelo de Holland (1997).

\section{Considerações Finais}

Os dados apresentados aportam evidências empíricas às proposições adlerianas sobre o comportamento vocacional, sugerindo que indivíduos de diferentes ordens de nascimento desenvolvem processos vocacionais distintos. Na família se desenvolvem os primeiros esforços dos indivíduos para encontrar um lugar significativo e uma identidade particular na coletividade humana. Cada pessoa busca esta identidade de uma forma particular, e a exploração das estratégias e trajetórias tomadas pelo indivíduo e por membros da família parece ser útil tanto para clientes em orientação de carreira quanto para os envolvidos em qualquer atividade com objetivos de insight e autoconhecimento. A teoria adleriana não faz predições sobre qual tipo de pessoa irá seguir determinada carreira, mas se propõe a identificar o percurso individual na busca por pertencer e ser valorizado com uma identidade única no contexto familiar e, a seguir, na sociedade e no mundo do trabalho. Sobre a ordem de nascimento, se um primogênito se desenvolve em uma direção específica, assumindo um papel, identidade e atributos determinados, os filhos subseqüentes provavelmente tomarão outras direções. Esta pode ser uma das razões pelas quais primogênitos tendem a alcançar maior status profissional, pois encontram desocupados os lugares mais privilegiados na família, suas realizações são mais efusivamente festejadas e são mais estimulados pelos pais.

É importante salientar que o uso adequado de informações sobre ordem de nascimento no aconselhamento vocacional requer que o profissional da orientação investigue como o indivíduo percebe o seu papel na família de origem. Ora, indivíduos de uma mesma posição na genitura familiar podem perceber de maneiras muito distintas a si mesmos e suas famílias. Portanto, embora favoreça determinadas tendências comportamentais, a posição ordinal deve ser contextualizada na experiência única de cada indivíduo. O impacto da ordem de nascimento está na dependência da interpretação particular que o sujeito dá ao seu contexto 
de vida. A psicologia adleriana se preocupa com o entendimento desta perspectiva fenomenológica única que a pessoa constrói a partir das suas primeiras experiências. E a ordem de nascimento é apenas uma das informações que podem ser úteis na busca desta compreensão. Os resultados deste estudo recomendam que pesquisadores e profissionais considerem o papel da ordem de nascimento no comportamento vocacional.

\section{Referências}

Adler, A. (1954). El carácter neurótico. Buenos Aires: Paidós. (Original publicado em 1912).

Adler, A. (1969). The science of living. New York: Anchor Books. (Original publicado em 1929).

Anastasi, A., \& Urbina, S. (2000). Testagem psicológica. Porto Alegre: Artes Médicas.

Ansbacher, H. L., \& Ansbacher, R. R. (1956). The individual psychology of Alfred Adler. New York: Harper and Row.

Balbinotti, M., Magalhães, M., Callegari, S., \& Fonini, C. R. (2004). Estudo fatorial exploratório da versão brasileira do inventário de preferências profissionais. In Sociedade Brasileira de Psicologia (Org.), Resumos da XXXIV Reunião Anual de Psicologia [CD-ROM]. Ribeirão Preto: SBP.

Baer, M., Oldham, G. R., Hollingshead, A. B., \& Jacobsohn, G. C. (2005). Revisiting the birth order-creativity connection: the role of sibling constellation. Creativity Research Journal, 17 (1), 67-77.

Bohmer, P., \& Sitton, S. (1993). The influence of birth order and family size on notable american women's selection of careers. Psychological Record, 43 (3), 124-132.

Bradley, R. W. (1984). Using sibling dyads to understand career development. The Personnel and Guidance Journal, 62 (2), 397-400.

Bradley, R. W., \& Mims, G. A. (1992). Using family systems and birth order dynamics as the basis for a college career decision-making course. Journal of Counseling and Development, 70 (2), 445-448.

Bryant, B. L. (1987). Birth order as a factor in the development of vocacional preferences. Individual Psychology, 48 (1), 36-41.

Chalfant, D. (1994). Birth order, perceived parental favoritism. And feelings toward parents. Individual Psychology, 50 (1), 52-57.

Clark, R. D., \& Rice, G. A., (1982). Family constellations and eminence: the birth orders of Nobel Prize winners. Journal of Psychology, 110 (1), 281-287.

Claxton, R. P. (1994). Empirical relationships between birth order and two types of parental feedback. Psychological Record, 44 (3), 475-487.
Dreikurs, R. (1963). Individual psychology: the Adlerian point of view. In J. M. Wepman \& R. Heine (Orgs.), Concepts of personality (pp.147-162). Chicago: Aldine.

Fakouri, E., \& Hafner, J. L. (1984). Early recollections of first-borns. Journal of Clinical Psychology, 40 (1), 209-213

Helson, R., \& Crutchfield, R.S. (1970). Mathematicians: The creative researcher and the average PhD. Journal of Consulting and Clinical Psychology, 34 (2), 250-257.

Holland, J. O. (1985). Making vocational choices: a theory of vocational personalities and workenvironments. Englewood Cliffs, NJ: Prentice-Hall.

Holland, J. O. (1997). Making vocational choices: a theory of vocational personalities and work environments. Odessa: PAR.

Leong, F. T, Hartung, P. J., Goh, D., \& Gaylor, M. (2001). Appraising birth order in career assessment: linkages to Holland's and Super's models. Journal of Career Assessment, 9 (1), 25-39.

Magalhães, M. (2005). Personalidades vocacionais e desenvolvimento na vida adulta: generatividade e carreira profissional. Tese de doutorado não-publicada, Universidade Federal do Rio Grande do Sul, Porto Alegre.

Magalhães, M., Balbinotti, M., \& Fisher, D. (2004). Interesses vocacionais de adolescentes e adultos no Rio Grande do Sul: diferenças relacionadas ao sexo. In Sociedade Brasileira de Psicologia (Org.), Resumos da XXXIV Reunião Anual de Psicologia [CD-ROM]. Ribeirão Preto: SBP.

Melillo, D. (1983). Birth order, perceived birth order, and family position of academic women. Individual Psychology, 39 (1), 57-62.

Nisbett, R. E. (1968). Birth order and participation in dangerous sports. Journal of Personality and Social Psychology, 9 (3), 352-353.

Roe, A. A. (1953). Psychological study of eminent psychologist and anthropologist and a comparison with biological and physical scientist. Psychological Monographs, 67 (1), $12-23$.

Savickas, M. L. (1998). Career style assessment and counseling. In T. Sweeney (Org.), Adlerian counseling: a practitioner's approach (pp.329-360). Philadelphia: Accelerated Development Press.

Schubert, D. S. P., Wagner, M. E., \& Schubert, H. J. P. (1977). Family constellation and creativity: first born predominance among classical music composers. Journal of Psychology, 95 (1), 147-149.

Skinner, N. F. (1997). Hypochondria in women as a function of birth order. Journal of the Royal Anthropological Institute, 3 (4), 1344-1346.

Simonton, D. K. (1999a). Genius, creativity and leadership: historiometric inquiries. Cambridge: Harvard University Press.

Simonton, D. K. (1999b). Origins of genius: Darwinian perspectives on creativity. Oxford: Oxford University Press.

Sohl, A.S., Yusuff, K.B. (1991). Birth order and participation in high and low dangerous Sports among selected Nigerian athletes. Journal of Asian and African Studies, 26 (3), 276-278. 
Sulloway, F. J. (1999a). Vocação rebelde:ordem de nascimento, dinâmica familiar e vidas criativas. São Paulo: Record.

Sulloway, F. J. (1999b). Birth order. In M. A. Runco (Ed.), Encyclopedia of creativity (pp.189-202). San Diego: Academic Press.

Super, D. E., Savickas, M. L., \& Super, C. (1996). Career choice and development. In D. Brown \& L. Brooks (Orgs.), The life-span, life-space approach to careers (pp.121-178). San Francisco: Jossey-Bass Publishers.

Terry, W. S. (1989). Birth order and prominence in the history of psychology. Psychological Record, 39 (3), 333-337.

Very, P. S., \& Prull, R. W. (1970). Birth order, personality development and the choice of law as a profession. Journal of Genetic Psychology, 116 (1), 119-221.

Watkins, C. E., Jr. (1984). The individual psychology of Alfred Adler: toward an Adlerian vocational theory. Journal of Vocational Behavior, 24 (1), 28-47.
Watts, R. E., \& Engels, D. W. (1995). The life task of a vocation: a review of Adlerian research literature. TCA Journal, 23 (1), 9-20.

Watkins, C. E. , Jr., \& Savickas, M. L. (1990). Psychodynamic career counseling. In W. B. Walsh \& S. H. Osipow (Orgs.), Career counseling: contemporary topics in vocational psychology (pp.79-116). Hillsdale, NJ: Lawrence Erlbaum Associates.

Zajonc, R. B. (2001). The family dynamics of intellectual development. American Psychologist, 56 (6/7), 490-496.

Zajonc, R. B., \& Mullally, P. R. (1997). Birth order: reconciling conflicting effects. American Psychologist, 52 (4), 685-699.

Recebido em: 29/8/2006

Aprovado em: 16/11/2006 